\title{
Avaliação de 67 casos de protrusão da glândula da terceira pálpebra em cães (2005-2010)
}

[Evaluation of 67 cases of prolapse of the third eyelid gland in dogs (2005-2010)]

\author{
R.V.R. Peixoto ${ }^{1}$, P.D. Galera ${ }^{2}$
}

\begin{abstract}
${ }^{1}$ Aluno de pós-graduação - Faculdade de Agronomia e Medicina Veterinária - Universidade de Brasília - Brasília, DF ${ }^{2}$ Faculdade de Agronomia e Medicina Veterinária - Universidade de Brasília - Brasília, DF
\end{abstract}

\begin{abstract}
RESUMO
Avaliaram-se 67 casos de protrusão da glândula da terceira pálpebra (cherry eye) em cães entre 2005 e 2010. Foram analisados a incidência da doença por gênero, a raça, a idade, o acometimento uni ou bilateral e a eficácia da técnica de Morgan pocket no reposicionamento da glândula. A protrusão da glândula da terceira pálpebra foi mais frequente em fêmeas $(62,6 \%)$, e a raça mais acometida foi Lhasa Apso, seguida de cães sem raça definida (SRD). A idade dos animais variou entre dois meses e 13 anos (média de três anos), sendo mais frequente em animais com idade abaixo de 24 meses. A afecção manifestou-se no olho direito de $30(44,8 \%)$ cães, no olho esquerdo de 18 (26,8\%), e, em 19 animais $(28,4 \%)$, a condição foi bilateral. Verificou-se que a técnica de Morgan pocket foi eficiente na resolução da afecção e requer mais cuidados no pós-operatório das raças grandes e gigantes.
\end{abstract}

Palavras-chave: cão, glândula da terceira pálpebra, cherry eye, protrusão, Morgan pocket

\begin{abstract}
In this study, 67 cases of dogs with prolapse of the third eyelid gland (cherry eye) between 2005 and 2010 were evaluated. The incidence of the disease by gender, age and breed was checked, and also if involvement was uni or bilateral and the effectiveness of the Morgan pocket technique of repositioning the gland. Prolapse of the third eyelid was more incident in females (62.6\%), and Lhasa Apso and mongrel dogs were the most affected breeds. The age of the animals ranged between two months and 13 years (average of three years), and cases were more frequent in animals younger than 24 months. The disease manifested itself in the right eye of 30 (44.8\%) dogs, in the left eye of 18 (26.8\%) dogs, and in 19 animals $(28.4 \%)$ the condition was bilateral. Morgan pocket technique was effective in the resolution of the complaint and demands more intensive care in post-surgery of large and giant breeds.
\end{abstract}

Keywords: dog, third eyelid, cherry eye, prolapse, Morgan pocket

\section{INTRODUÇÃO}

A terceira pálpebra ou membrana nictitante é uma estrutura de proteção móvel, composta por uma cartilagem na forma de $\mathrm{T}$, pela glândula da terceira pálpebra, pelas conjuntivas bulbar e palpebral e por folículos linfoides que se localizam sobre a superfície bulbar (Moore e Constatinescu, 1997; Slatter, 2005).

A base da cartilagem e a glândula são mantidas na sua posição anatômica por um retináculo fascial pobremente definido para a região do músculo oblíquo ventral e a do reto medial (Ward e Hendrix, 2003). A terceira pálpebra pode sediar afecções, como a eversão da cartilagem da terceira pálpebra e neoplasias. No entanto, a afecção mais comum nesta estrutura é a protrusão da glândula da terceira pálpebra, mais comumente relatada em cães (Slatter, 2005; Hendrix, 2007), embora tenha sido descrita em gatos (Chahory et al., 2004).

Recebido em 25 de maio de 2011

Aceito em 30 de maio de 2012

E-mail: paulaeye@unb.br 
A etiologia dessa condição ainda não foi claramente elucidada, mas acredita-se que a protrusão da glândula decorra de uma deformidade no retináculo que liga a glândula à periórbita, levando a uma frouxidão deste tecido (Slatter, 2005; Hendrix, 2007). Geralmente manifesta-se em animais jovens (Delgado, 2005; Hendrix, 2007) e algumas raças são descritas como predispostas, a exemplo do Beagle (Dugan et al., 1992; Hendrix, 2007), Cocker Spaniel Americano, Lhasa Apso, Pequinês (Hendrix, 2007) e Bulldog Inglês (Hendrix, 2007; Galera et al., 2009).

O tratamento é exclusivamente cirúrgico (Peixoto e Galera, 2009). Há alguns anos propôsse a excisão completa ou parcial da parte prolapsada (Barnett, 1978), procedimento não recomendado, pois a excisão da glândula pode, sabidamente, desencadear a ceratoconjuntivite seca iatrogênica (Almeida et al., 2004; Peixoto e Galera, 2009; Galera et al., 2010). Como alternativa à excisão parcial ou total da glândula da terceira pálpebra, foram desenvolvidas técnicas cirúrgicas, a exemplo da ancoragem da glândula na periórbita (Blogg, 1980; Albert et al., 1982; Gross, 1983; Kaswan e Martin, 1985; Stanley e Kaswan, 1994) e da criação de uma bolsa conjuntival para sepultar a glândula (Moore, 1983; Morgan et al., 1993).

Objetivou-se avaliar 67 casos de protrusão da glândula da terceira pálpebra quanto à raça, ao sexo e à idade dos cães acometidos, se o acometimento foi uni ou bilateral, bem como os resultados obtidos com o reposicionamento da glândula da terceira pálpebra.

\section{MATERIAIS E MÉTODOS}

Realizou-se um levantamento do prontuário de 900 animais atendidos no Serviço de Oftalmologia Veterinária (2005-2010), dos quais 67 apresentaram diagnóstico de protrusão da glândula da terceira pálpebra, descartando-se o diagnóstico diferencial de eversão da cartilagem desta estrutura. Todos os animais foram submetidos ao exame oftálmico de rotina, compreendendo teste da lágrima de Schirmer-1 (Teste de Schirmer®- Ophthalmos - São Paulo SP) (TLS-1), teste da fluoresceína (Fluoresceína Strips® - Ophthalmos - São Paulo - SP), biomicroscopia em lâmpada de fenda (Portable Slit lamp SL-15® - Kowa - Califórnia - EUA), aferição da pressão intraocular pela tonometria de aplanação (Tonopen® XL - Medtronic) e inspeção da superfície bulbar da terceira pálpebra mediante instilação tópica de colírio anestésico (Anestésico® - Allergan Produtos Farmacêuticos Ltda. - Guarulhos - SP).

Recomendou-se reposicionamento cirúrgico da glândula a todos os cães, mas apenas 41 proprietários aderiram ao tratamento proposto. Os animais foram submetidos à anestesia geral inalatória e posterior antissepsia local padrão. Seguiu-se a cirurgia para a correção da protrusão da glândula da terceira pálpebra, empregando-se a técnica de Morgan pocket (Morgan et al., 1993), com fio poliglactina 910, encastoado de fábrica (Vycril@ - Ethicon - São José dos Campos - SP), calibre 5-0 ou 6-0, conforme o tamanho do animal. No pós-operatório imediato, administrou-se, por via subcutânea, cetoprofeno (Ketofen® - Merial Saúde Animal Ltda. - SP), na dose única de $2 \mathrm{mg} / \mathrm{kg}$. Prescreveu-se o uso de colar elisabetano e a utilização de ciclosporina tópica a 0,2\% (Optimmune® - Schering-Plough - São Paulo - SP) em intervalos de oito horas durante 10 dias consecutivos.

Os animais foram acompanhados aos 10 dias de pós-operatório e, posteriormente, em avaliação tardia, a qual variou entre quatro e 36 meses, para verificação dos resultados decorrentes da cirurgia e aferição da produção lacrimal. Todas as cirurgias foram realizadas pelo mesmo cirurgião.

\section{RESULTADOS E DISCUSSÃO}

Foram atendidos 67 cães com protrusão da glândula da terceira pálpebra, perfazendo $7,4 \%$ dos 900 casos atendidos. Destes, 42 eram fêmeas $(62,7 \%)$ e 25 machos $(37,3 \%)$, com idade variando de dois meses a 13 anos (média de 33 meses), sendo que 64,2\% (43/67) dos animais acometidos apresentavam idade abaixo de dois anos, confirmando relatos anteriores (Delgado, 2005; Hendrix, 2007). A afecção manifestou-se no olho direito de $30(44,8 \%)$ cães, no olho esquerdo de 18 (26,8\%), e, em 19 animais $(28,4 \%)$, a condição foi bilateral. No conhecimento dos autores, este é o primeiro trabalho a relatar maior predisposição de fêmeas, pois não há descrição quanto à predileção por gênero. Entretanto, há referência de que as fêmeas são mais susceptíveis ao 
desenvolvimento da ceratoconjuntivite seca (Sanchez et al., 2007) e que possuem a glândula da terceira pálpebra menos espessa (Cabral et al., 2005). A literatura não estabelece se a condição é mais evidente de forma uni ou bilateral e quanto a um olho ser mais afetado que outro (Slatter, 2005; Hendrix, 2007); no entanto, neste estudo observou-se que, na condição unilateral, houve maior acometimento do olho direito em relação ao esquerdo.

As raças mais acometidas, por ordem decrescente, foram Lhasa Apso com 12 casos $(17,9 \%)$; SRD com sete casos $(10,4 \%)$; Beagle e Shar Pei compreendendo seis casos cada (9\%); Cocker Spaniel Americano e Shih Tzu com cinco casos cada (7,5\%); Bulldog Inglês e Poodle com quatro casos cada $(5,97 \%)$; Bulldog Francês e Mastino Napoletano com três casos cada $(4,5 \%)$; Basset Hound, Dachshund e Fila Brasileiro com dois casos cada (3\%) e Boxer, Maltese, Mastiff, Rottweiler, Weimaraner e Yorkshire Terrier com um caso cada $(1,5 \%)$. As raças Beagle (Dugan et al., 1992; Hendrix, 2007), Cocker Spaniel Americano e Lhasa Apso (Hendrix, 2007) são consideradas susceptíveis ao cherry eye (Morgan et al., 1993; Severin, 1996; Moore Constantinescu, 1997), mas deve-se destacar a descrição desta afecção em cães SRD e elevada frequência no Shar Pei, com a mesma proporção de raças consideradas predispostas, como o Beagle. Ainda, cães das raças Fila Brasileiro, Dachshund, Boxer e Rotweiller não são descritos como predispostos à protrusão da glândula da terceira pálpebra. Inicialmente, pensou-se atribuir a ocorrência da afecção nos cães SRD à casuística do serviço, o que suscitou o levantamento do número de cães com e sem raça definida. Verificou-se, então, que apenas 10,8\% dos casos atendidos no setor eram SRD.

O primeiro a descrever o sepultamento da glândula foi Moore (1983). Morgan et al. (1993) modificaram a técnica, denominando-a Morgan pocket (Moore et al., 1994). Recentemente, Plummer et al. (2008) relataram a ancoragem da glândula na cartilagem da terceira pálpebra, sem alterar sua mobilidade e com bom índice de sucesso, aumentado as opções dos cirurgiões. Dos 41 animais operados, 11 apresentavam acometimento bilateral, sendo a cirurgia realizada em 52 olhos. Embora a formação de cistos seja descrita como eventual complicação da técnica (Hendrix, 2007; Plummer et al.,
2008), em nenhum dos 52 olhos operados observou-se tal intercorrência.

Nove olhos $(17,3 \%)$ de nove animais diferentes apresentaram recidiva, observando-se, então, sucesso cirúrgico em $82,7 \%$ dos casos. Dentre as recidivas, conforme relato de proprietários, cinco cães não tiveram pós-operatório adequado, pois os animais apresentavam temperamento indócil ou agitado. Estas cinco recidivas ocorreram em uma fêmea da raça Mastiff aos 11 dias de pósoperatório, uma fêmea da raça Mastino Napoletano aos oito dias de pós-operatório, um macho da raça Rottweiler aos 15 dias de pósoperatório, um macho da raça Fila Brasileiro aos quatro dias de pós-operatório e uma fêmea da raça Lhasa Apso aos dois dias de pós-operatório. Seus proprietários relataram que os animais quebraram o colar elisabetano e "coçaram" o olho, levando à inflamação exacerbada da glândula da terceira pálpebra, sinais clínicos de infecção palpebral e deiscência da sutura. Nenhum proprietário afirmou ter retirado o colar elisabetano. Ainda, pode-se inferir que o calibre do fio para as raças gigantes deva ser maior. A opção pelos calibres 5,0 e 6,0 deve-se à padronização da técnica, conforme descrita em literatura e com intenção de se minimizarem as variáveis do estudo.

Relativamente às demais recidivas, um macho da raça Shar Pei apresentou inflamação intensa no $14^{\circ}$ dia de pós-operatório cursando com deiscência de sutura, tendo recebido antibioticoterapia tópica (pomada de cloranfenicol). Os outros três animais, uma fêmea da raça Basset Hound, um macho da raça Beagle e uma fêmea da raça Cocker Spaniel Americano, cursavam com intensa inflamação ocular decorrente de ceratoconjuntivite seca, previamente à cirurgia, e apresentaram recidiva da doença com oito, nove e 15 dias de pós-operatório, respectivamente. Verificou-se, portanto, um bom índice de sucesso cirúrgico, mas o seu êxito, assim como nos demais procedimentos cirúrgicos, é amplamente dependente do período pós-operatório. Um pósoperatório com muita inflamação, na maioria das vezes decorrente da cronicidade da afecção, e com constantes lesões à terceira pálpebra, eleva a possibilidade de recidiva (Plummer et al., 2008). Excluindo-se problemas assumidos pelos proprietários quanto ao pós-operatório, a taxa de sucesso cirúrgico foi de $92,3 \%$, valor próximo ao 
descrito por Morgan et al. (1993) e superior ao descrito por Delgado (2005).

Mesmo diante de tais intercorrências, a técnica de Morgan pocket foi eficiente com relação ao reposicionamento da glândula da terceira pálpebra. Em um trabalho citado como referência aos demais, Morgan et al. (1993) atribuíram êxito cirúrgico em $94,1 \%$ dos casos. Ressalta-se, entretanto, que dos 89 casos reportados por esses autores, apenas 17 olhos foram operados. Posteriormente, Delgado (2005) obteve resultados cirúrgicos favoráveis em $87 \%$ dos casos. No entanto, raças grandes a gigantes, como Mastiff, Mastino Napoletano, Rottweiler ou Fila Brasileiro, não estavam entre os animais operados por Morgan et al. (1993), tampouco houve relatos quanto a complicações do pósoperatório e ao temperamento dos animais, como observado neste trabalho.

Trabalhos comparativos entre as técnicas cirúrgicas de ancoragem da glândula ou da criação de uma bolsa conjuntival ainda são escassos e imprecisos, e seu êxito varia com a aptidão do cirurgião (Hendrix, 2007).

A escolha da técnica adequada implica lidar com aspectos vantajosos e adversos. Plummer et al. (2008) relatam que cães submetidos à ancoragem da glândula frequentemente apresentam problemas no correto funcionamento da terceira pálpebra, restringindo sua movimentação, o que não ocorre quando se empregam técnicas de sepultamento da glândula. A opção pelo emprego da técnica de Morgan pocket deveu-se a este aspecto e à sua exequibilidade. A movimentação da terceira pálpebra manteve-se íntegra nos 41 animais deste estudo.

O pós-operatório compreende a utilização de antibióticos e corticoides tópicos (Slatter, 2005). Entretanto, neste estudo, optou-se pela utilização da ciclosporina a $0,2 \%$, e não de antiinflamatório esteroide, dado o conhecido efeito anti-inflamatório da ciclosporina (Herrera, 2008), bem como a segurança de sua utilização em casos de ceratites ulcerativas (Giuliano e Moore, 2007) desencadeadas por eventual automutilação no pós-operatório imediato. Tal fato foi observado em alguns animais deste estudo, a despeito do uso do colar elisabetano. Por se tratar de cirurgia limpa, dispensou-se a antibioticoterapia, sem serem observados sinais de infecção cirúrgica. Restrições foram feitas aos casos de recidiva, que tiveram que ser reavaliados e submetidos a um segundo procedimento.

Avaliaram-se animais com período excedente a 30 meses de pós-operatório, verificando-se resultados clínicos e cirúrgicos favoráveis, além do efeito cosmético obtido. A opção pela retirada da glândula não é comum na literatura (Almeida et al., 2004; Sanchez et al., 2007; Galera et al., 2010) nem deve ser justificada em razão de insucessos ocorridos devido às técnicas preconizadas. Fatores como a destreza cirúrgica e a compreensão de um pós-operatório adequado, bem como a escolha dos pacientes, também estão relacionados à resolução favorável dos casos.

\section{CONCLUSÕES}

Esta é a primeira descrição da protrusão da glândula da terceira pálpebra em cães sem raça definida, e da predisposição por fêmeas na literatura consultada, assim como do olho direito. O reposicionamento da glândula da terceira pálpebra, realizado com acurácia e associado a pós-operatório adequado, resulta em sucesso no tratamento do cherry eye, motivo pelo qual deve ser incentivado. A escolha adequada do paciente cirúrgico pode reforçar os resultados favoráveis. Entretanto, mesmo com as dificuldades do pósoperatório de animais de raças grandes e gigantes, a cirurgia deve ser realizada por ser este o tratamento de eleição dessa afecção, mas requer mais cuidados no pós-operatório destes cães.

\section{REFERÊNCIAS}

ALBERT, R.A.; GARRETT, P.D.; WHITLEY, R.D. et al. Surgical correction of everted in two cats. J. Am. Vet. Med. Assoc., v.180, p.763-766, 1982.

ALMEIDA, D.E.; MAMEDE, F.V; ORTIZ, J.P.D. et al. Iatrogenic keratoconjunctivitis sicca in a dog. Cienc. Rural., v.34, p.921- 924, 2004.

BARNETT, K.C. Diseases of the nictitating membrane of the dog. J. Small Anim. Pract., v.19, p.101-108, 1978.

BLOGG, J.R. Diseases of the Eyelids. In: BLOGG J.R. (Ed). The Eye in Veterinary Practice: Extraocular Disease. Philadelphia: W.B. Saunders, 1980. p.342. 
CABRAL, V.P.; LAUS, J.L.; DAGLI, M.L.Z. et al. Canine lacrimal and third eyelid superficial glands' macroscopic and morphometric characteristics. Cienc. Rural., v.35, p.391-397, 2005.

CHAHORY, S.; CASTRA, M.; TRIO, S. et al. Tree cases of prolapse of the nictitans gland in cats. Vet. Ophtalmol., v.7, p.417-419, 2004.

DELGADO, E. Recolocação cirúrgica da glândula da membrana nictitante em canídeos pela técnica de bolsa conjuntival - 23 casos clínicos. Rev. Port. Ciênc. Vet., v.100, p.89-94, 2005.

DUGAN, S.J.; SEVERIN, G.A.; HUNGERFORD, L.L. et al. Clinical and histologic evaluation of the prolapsed third eyelid gland in dogs. J. Am. Vet. Med. Assoc., v.15, p.1861-1867, 1992.

GALERA, P.D.; FALCÃO, M.S.; CASTELLON, M.F.L.F. Particularidades oftálmicas das raças braquicefálicas. MEDVEP. Rev. Cient. Med. Vet. Peq. Anim. Anim. Estim., v.7, p.80-88, 2009.

GALERA, P.D.; YASUNAGA, K.L., PEIXOTO, R.V.R. Ceratoconjuntivite seca iatrogênica. MEDVEP. Rev. Cient. Med. Vet. Peq. Anim. Anim. Estim., v.8, p.456-459, 2010.

GIULIANO, E.A.; MOORE, C.P. Diseases and surgery of the lacrimal and secretory sistem. In: GELLAT, K.N. Veterinary Ophthalmology. 4.ed. Philadelphia: Lippincott Williams \& Wilkins, 2007. ch.13. p.633-661.

GROSS, S.L. Effectiveness of a Modification of the Blogg technique for replacing the prolapsed gland of the third eyelid. Am. Col. Vet. Ophthal., v.14, p.38-42, 1983.

HENDRIX, D.V.H. Canine Conjunctiva and Nictitating Membrane. In: GELLAT, K.N. Veterinary Ophthalmology. 4. ed. Philadelphia: Lippincott Williams \& Wilkins, 2007. ch.14. p.662-689.

HERRERA, D. Afecções da Córnea. In: Oftalmologia Clínica em Animais de Companhia 1.ed. São Paulo: MedVet, 2008. cap. 7, p.117-122.

KASWAN, R.L.; MARTIN, C.L. Surgical correction of third eyelid prolapse in dogs. J. Am. Vet. Med. Assoc., v.186, p.83, 1985.

MOORE, C.P. Alternative technique for prolapsed gland of the third eyelid (replacement technique). In: BOJRAB, M.J. (Ed). Current Techniques in Small Animal Surgery. Philadelphia: Lea \& Febiger, 1983. p.52-53.
MOORE, C.P.; FRAPPIER, B.L.; LINTON, L.L. Effects of two surgical replacement techniques on excretory ducts of normal canine third eyelid glands. Am. Col. Vet. Ophthal., v.25, p.25, 1994.

MOORE, C.P.; CONSTATINESCU, G.M. Surgery of the adnexa surgical management of ocular disease. Vet. Clin. North Am.: Small Anim. Pract., v.27, p.1011-1066, 1997.

MORGAN, R.V.; DUDDY, J.M.; McCLURG, K. Prolapse of the gland of the third eyelid in dogs: a retrospective study of 89 cases (1980 to 1990). J. Am. Anim. Hosp. Assoc., v.29, p.56-60, 1993.

PEIXOTO, R.V.R.; GALERA, P.D. Revisão de literatura: técnicas cirúrgicas para redução da protrusão da glandula da terceira pálpebra em cães. MEDVEP. Rev. Cient. Med. Vet. Peq. Anim. Anim. Estim., v.7, p.319-322, 2009.

PLUMMER, C.E.; KÄLLBERG, M.E.; GELATT, K.N. et al. Intranictitans tacking for replacement of prolapsed gland of the third eyelid in dogs. Vet. Ophthalmol., v.11, p.228-233, 2008.

SANCHEZ, R.F.; INNOCENT, I.; MOULD, J. et al. Canine keratoconjunctivitis sicca: diseases trends in a review of 229 cases. J. Small Anim. Pract., v.48, p.211-217. 2007.

SEVERIN, G.A. Severin's Ophthalmology Notes. 3.ed. Ft. Collins: C.O. Veterinary Ophthalmology Notes. v.214, 1996. 546p.

SLATTER, D. In:___ Fundamentos da Oftalmologia Veterinária. 3.ed. São Paulo: Roca, 2005. 671p.

STANLEY, R.G.; KASWAN, R.L. Modification of the orbital rim anchorage method for surgical replacement of the gland the third eyelids in dogs. $J$. Am. Vet. Med. Assoc., v.205, p.1412-1414, 1994.

WARD, D.A.; HENDRIX, D.V.H. Doenças e cirurgias dos sistemas lacrimal e nasolacrimal no cão. In: GELATT, K.N. Manual de Oftalmologia Veterinária. 3.ed. Barueri-SP: Manole, 2003. cap.4, p.76-87. 